\title{
Caracterización de Tagetes filifolia del centro-sur de México por morfometría de los aquenios
}

\section{Characterization of Tagetes filifolia from central-south Mexico by morphometric achene traits}

\author{
Carlos A. Núñez-Colín ${ }^{1 *}$, Miguel A. Serrato-Cruz², María Teresa Santos-Cortés², César del C. Luna-Morales², \\ Juan Martínez-Solís² y Jesús Axayácatl Cuevas-Sánchez ${ }^{2}$ \\ ${ }^{I}$ Campo Experimental Bajio, Instituto Nacional de Investigaciones Forestales, Agricolas y Pecuarias. Km. 6.5 Carretera Celaya-San Miguel de Allende, \\ Apartado postal 112, 38010 Celaya, Guanajuato, México. \\ ${ }^{2}$ Departamento de Fitotecnia, Universidad Autónoma Chapingo. Km. 38.5 Carretera México-Texcoco, 56230, Chapingo, Estado de México, México. \\ *Correspondencia: lit007a@gmail.com
}

\begin{abstract}
Resumen. Tagetes filifolia Lag. es una especie ruderal ampliamente distribuida en México de la cual se empieza a conocer su variabilidad morfológica, como parte de una serie de estudios relacionados con su posible domesticación para obtener biopesticidas. Para ampliar el conocimiento sobre este recurso vegetal se definieron mediante 11 características del aquenio 104 recolecciones del centro-sur de México empleando una técnica digital de imágenes. Se identificaron 5 diferentes grupos morfológicos. El Grupo I presentó recolecciones con aquenios pequeños y anchos; el Grupo II, aquenios grandes y anchos; el Grupo III, aquenios pequeños y alargados; el Grupo IV, aquenios grandes y alargados, y el Grupo V, aquenios grandes pero más alargados que los del Grupo IV. Las variables más discriminantes fueron el índice de alargamiento, índice de compactación, índice de redondez, relación eje menor/ eje mayor para la raíz canónica 1, y perímetro longitudinal y longitud del eje mayor para la raíz canónica 2. La gran variabilidad de las características de la forma y tamaño de los aquenios de T. filifolia se pudo agrupar en 5 morfotipos, los cuales no estuvieron presentes en toda la región centro-sur de México.
\end{abstract}

Palabras clave: Asteraceae, Tageteae, variabilidad del aquenio, morfología, análisis multivariado.

\begin{abstract}
Tagetes filifolia Lag. is a ruderal species widely distributed in Mexico from which is starting to make knowledge about its morphological variability as a part of a study sequence related with its possible domestication to obtain biopesticides. For acquiring more knowledge on this plant resource, 104 accessions from central-south Mexico were morphologically characterized by 11 achene traits employing a digital images technique. Five different morphologic groups were identified. Group I had accessions with small and wide achenes, group II had big and wide achenes, group III had small and long achenes, group IV had big and long achenes, and group V had big achenes but longer than group IV. The most discriminant variables were elongation index, compactness index, roundness index, minor axis length/ major axis length ratio to get the canonical root 1 , and longitudinal perimeter and major axis length to get the canonical root 2. The variability in shape and size of $T$. filifolia achenes was grouped in 5 morphotypes, however not all morphotypes were present in all the central-south region of Mexico.
\end{abstract}

Key words: Asteraceae, Tageteae, achene variability, morphology, multivariate analysis.

\section{Introducción}

El anís de monte o anisillo Tagetes filifolia Lag. (Asteraceae: Tageteae), es un recurso fitogenético utilizado en medicina, alimentación y agricultura (Serrato y Quijano, 1994). En la actualidad representa una opción promisoria como insecticida y repelente para el control de insectos, como pulgones, trips o mosquitas blanca (Serrato et al., 2003; Camarillo-de la R. et al., 2007; Reyes-T et al., 2007),

Recibido: 21 abril 2009; aceptado: 17 agosto 2010 por lo que los trabajos que se realicen para su domesticación son importantes. La especie es de condición ruderal, viaria o arvense, tiene una amplia distribución geográfica en México (Neher, 1965; Turner, 1996; Villarreal, 2003), pero la información disponible en herbarios nacionales o en publicaciones sobre aspectos de su variabilidad es incompleta. La primera aproximación sobre la variabilidad de la especie es de Serrato-Cruz y Barajas-Pérez (2006), quienes describieron la morfología de algunos caracteres para 102 recolecciones in situ pertenecientes a la región centro-sur de México; mediante el análisis multivariado de características de los aquenios y del involucro, estos autores distinguen 6 grupos morfológicos, definidos 
principalmente por la longitud de arista, así como por la longitud y anchura de la semilla, aunque en especies domesticadas de Tagetes, las dimensiones del involucro tienen más importancia para la diferenciación morfológica (Serrato-Cruz et al., 1998), lo cual resalta el alto valor que tienen las características de la cabezuela en el estudio de variabilidad en Tagetes.

La forma y el tamaño de la semilla son características de la morfología reproductiva que han resultado de utilidad para el estudio de la variabilidad biológica de algunas especies de Asteraceae, variabilidad que, a su vez, es resultado de la adaptación a la diversidad ambiental en la cual se desarrollan (Flann et al., 2002; Fici y Presti, 2003); tales características morfológicas podrían ayudar a comprender más la variabilidad del aquenio, previamente detectada en T. filifolia (Serrato-Cruz y Barajas-Pérez, 2006).

La información antecedente sobre la longitud y anchura del aquenio de $T$. filifolia de diferente origen geográfico (Serrato-Cruz y Barajas-Pérez, 2006) da una idea parcial de la forma y tamaño de ese órgano reproductivo; sin duda, otras características especiales de esta estructura, incluso internas, podrían captarse mediante técnicas de morfometría basadas en digitalización de imágenes para detallar estructuras minúsculas, tal como se ha hecho en propágulos de origen sexual de algunas especies (González-Andrés y Ortiz, 1995; Nieto-Ángel et al., 2009).

Considerando que el conocimiento detallado de la forma y tamaño del aquenio de T. filifolia podría contribuir al planteamiento de otros trabajos sobre la relación biológico-ambiental de la especie, el objetivo de la presente investigación consistió en caracterizar, mediante morfometría digital, la variabilidad morfológica de los aquenios de 104 accesiones de T. filifolia de la región centro-sur de México.

\section{Materiales y métodos}

De septiembre a noviembre de 2004 se realizó una exploración botánica en distintas regiones de los estados de Guerrero, Jalisco, México, Michoacán, Oaxaca, Puebla y Veracruz para obtener muestras de los aquenios de capítulos de al menos 25 plantas de T. filifolia en cada sitio (1 000 a 3000 aquenios por muestra). Los lugares de recolección se ubicaron mediante un geoposicionador global Etrex, marca Garmín, registrando coordenadas geográficas y altitud (Apéndice 1). Las 104 muestras recolectadas se depositaron y registraron con las claves FIL001 a FIL104 en el Banco de Germoplasma Salvador Miranda Colín de la Universidad Autónoma Chapingo

De cada accesión se tomaron al azar 10 aquenios para ser digitalizados, uno por uno, con un scanner Genius
Color Page Vivid Pro II Film. A partir de las imágenes obtenidas y mediante el programa Image Tool versión 3 (Wilcox et al., 1995), se evaluaron las variables siguientes: perímetro longitudinal, área longitudinal, diámetro Feret

(de acuerdo con la fórmula $D F=\sqrt{(4 \cdot \text { Area }) / \pi} \quad$ ), longitud

del eje mayor, longitud del eje menor, índice de alargamiento (de acuerdo con la fórmula

$$
E I=\stackrel{\text { Longitud_del_eje_mayor }}{\text { Longitud_del_eje_menor }),}
$$

índice de redondez (de acuerdo con la fórmula $R I$ $=(4 \cdot \pi \cdot$ Area $) /$ Perimeter $\left.^{2}\right)$, índice de compactación (de acuerdo con la fórmula

$$
C I=D F \quad \text { Longitud_del_eje_mayor ) }
$$

y relación longitud del eje menor / longitud eje mayor (relación inversa al índice de alargamiento), longitud de la arista larga y longitud de la arista corta (Fig.1). Estas variables presentan una distribución normal y no existen variables autocorrelacionadas.

Se utilizó un análisis de agrupamiento (cluster) mediante la distancia euclidiana y el método de aglomeración de varianzas mínimas de Ward (Ward, 1963). Para determinar el número de grupos morfológicamente homogéneos en el dendrograma se realizó una prueba de partición mediante el criterio cúbico de agrupación (Johnson, 1998).

A partir de estos grupos, se realizó un análisis discriminante canónico. Las variables discriminantes en la formación de los grupos que se otuvieron en el dendrograma se identificaron en la estructura canónica total. Para corroborar los agrupamientos se proyectaron en las primeras raíces canónicas. Además se realizó un análisis de resubstitución (o análisis a posteriori) para identificar si las recolecciones fueron o no mal agrupadas por el análisis de agrupamiento, y se realizó la prueba de la distancia de Mahalanobis para verificar si los grupos son diferentes (Johnson, 1998). Todos los análisis se realizaron con el programa SAS, versión 8 (SAS Institute, 1999).

\section{Resultados}

De acuerdo con el criterio cúbico de agrupación (Fig. 2), en el dendrograma se identificaron 5 conjuntos morfológi- 

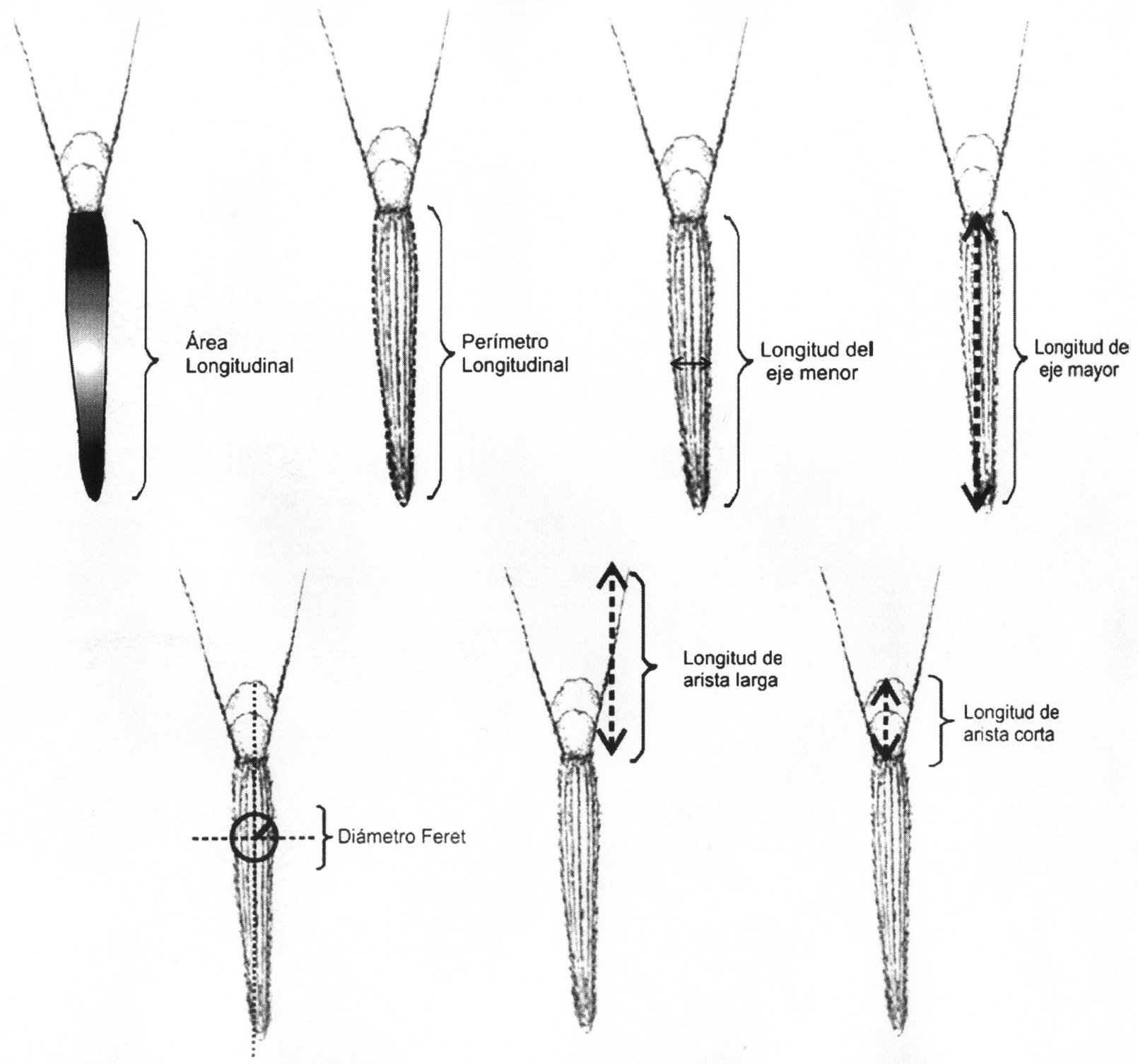

Figura 1. Variables morfométricas obtenidas de aquenios de Tagetes filifolia.

camente diferentes que se denominaron grupos (Fig. 3). En el análisis canónico se encontró que las 2 primeras raíces canónicas explicaron más del $78 \%$ de la variabilidad morfológica (Cuadro 1). Las variables más discriminantes para la raíz canónica 1 (Can 1) fueron el índice de alargamiento, la relación eje menor/eje mayor y los índices de redondez y de compactación, es decir, caracteres referentes a forma. Para la función canónica 2 (Can 2), fueron el perímetro longitudinal y la longitud del eje mayor del aquenio, es decir, caracteres referentes a tamaño (Cuadro 2).

En la proyección de las recolecciones en variables canó- nicas, el Can 1 permitió discriminar aquenios alargados (delgados) y casi redondos (anchos); mientras que el Can 2 facilitó la identificación de aquenios grandes y chicos (Fig. 4).

Las recolecciones del Grupo V se diferenciaron de los otras, principalmente por el índice de alargamiento (12.5), pero con menores índices de redondez $(0.23)$ y de compactación (0.33) y menor relación eje menor /eje mayor (0.08); el perímetro longitudinal y la longitud del eje mayor, tuvieron las mayores dimensiones $(13.4 \mathrm{~mm}$ y $6.1 \mathrm{~mm}$, respectivamente). Las recolecciones de este grupo presentaron aquenios grandes y muy delgados (Fig. 4). 
Cuadro 1. Valores propios de las raíces canónicas y estructura canónica total del análisis discriminante canónico de las características del aquenio de 104 colectas de T. filifolia de la región centro-sur de México

\begin{tabular}{lcc}
\hline \multirow{2}{l}{ Variable } & \multicolumn{2}{c}{ Raices canónicas } \\
& Can 1 & Can 2 \\
\hline Valor propio & 4.167 & 1.589 \\
Varianza aportada & 0.570 & 0.217 \\
Varianza acumulada & 0.570 & 0.787 \\
\hline Área longitudinal del aquenio & -0.407 & 0.777 \\
Perímetro longitudinal del aquenio & 0.182 & $\mathbf{0 . 9 5 1}$ \\
Longitud del eje mayor del aquenio & 0.308 & $\mathbf{0 . 9 1 5}$ \\
Longitud del eje menor del aquenio & -0.834 & 0.499 \\
Relación Eje menor/Eje mayor & $\mathbf{- 0 . 9 4 2}$ & -0.050 \\
Índice de alargamiento del aquenio & $\mathbf{0 . 9 6 4}$ & 0.053 \\
Índice de compactación del aquenio & $\mathbf{- 0 . 9 1 6}$ & -0.290 \\
Índice de redondez del aquenio & $\mathbf{- 0 . 9 2 3}$ & -0.260 \\
Diámetro Feret del aquenio & $\mathbf{- 0 . 4 4 6}$ & 0.839 \\
Longitud de arista corta & 0.267 & 0.373 \\
Longitud de arista larga & 0.220 & 0.428 \\
\hline
\end{tabular}

Las recolecciones de los Grupos III y IV presentaron índices intermedios de redondez (0.31) y compactación (0.39-0.40), y de relación eje menor/eje mayor (0.12-0.13); las del Grupo III presentaron menor longitud del eje mayor $(4.7 \mathrm{~mm})$ y de perímetro longitudinal (10.5) que las del Grupo IV en el que estas características presentaron una mayor expresión (5.7 $\mathrm{mm}$ y $12.9 \mathrm{~mm}$, respectivamente). Las del Grupo III tuvieron mayor índice de alargamiento (8.8) y en el Grupo IV ese índice fue menor (7.5). En el Grupo IV los aquenios fueron pequeños y delgados; en el Grupo III son grandes y tendentes a delgados (Fig. 4).

Los Grupos I y II presentaron los aquenios anchos (tendencia a redondo); pero en el Grupo I la longitud del eje mayor $(4.6 \mathrm{~mm})$ y el perímetro longitudinal $(10.6 \mathrm{~mm})$ resultaron menores que en el Grupo II (5.1 y $11.8 \mathrm{~mm}$, respectivamente). Los aquenios del Grupo II fueron grandes y anchos; los del Grupo I, pequeños y anchos (Fig. 4). Hay grupos morfológicos, como I, II y IV, que se distribuyen en toda la región centro-sur de México (Cuadro 3). El grupo

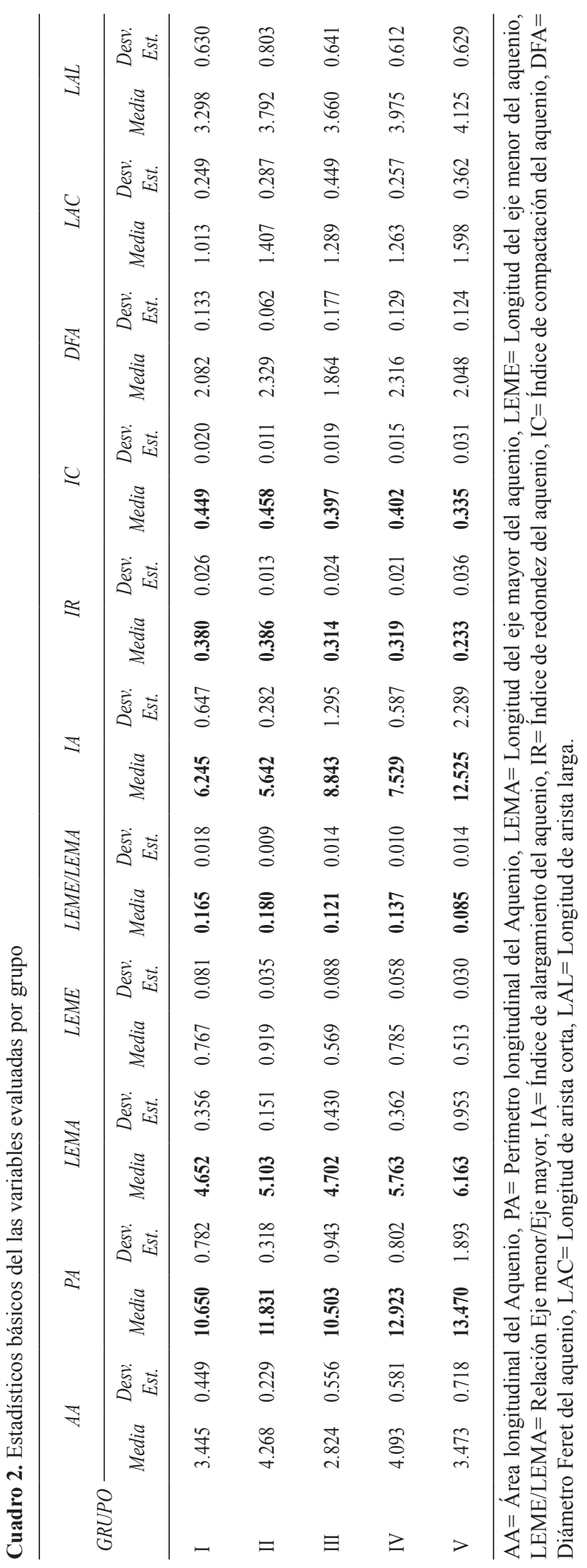




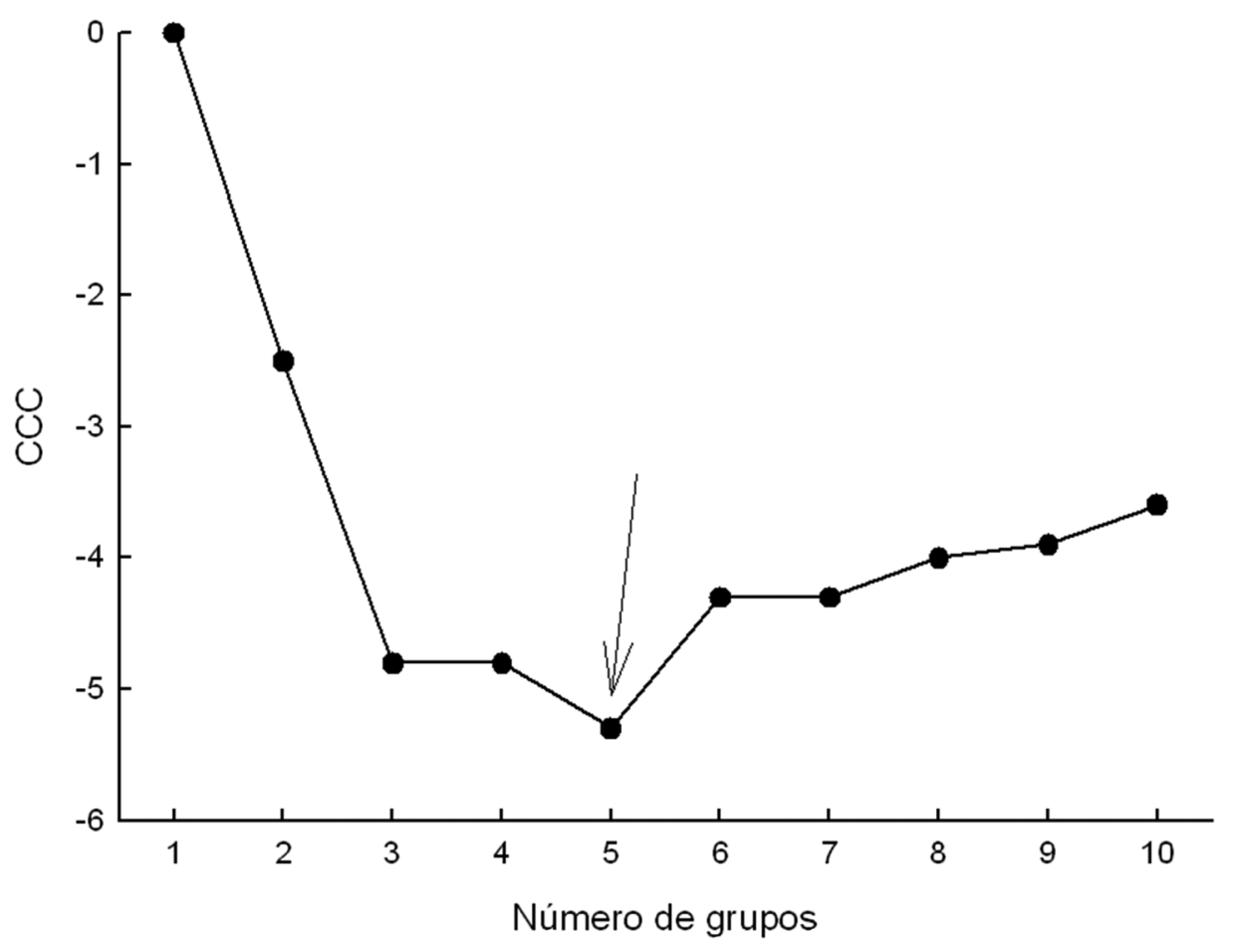

Figura 2. Criterio cúbico de agrupación (CCC) como prueba de partición, el número menor en la gráfica es el número correcto de agrupaciones.
$\mathrm{V}$ es una forma rara, y sólo se registró en 4 recolecciones. mientras que en el estado de Michoacán se pueden encontrar los 5 grupos morfológicos.

El análisis de resubstitución mostró pocos errores de clasificación en los grupos I, III y V (Cuadro 4), pero la prueba de Mahalanobis indicó que los 5 grupos morfológicos de T. filifolia eran diferentes (Cuadro 5).

\section{Discusión}

Las características de forma (índice de alargamiento de 5.6 a 12.5 e índice de redondez de 0.23 a 0.38 ) y de tamaño (de 4.6 a $6.1 \mathrm{~mm}$ ) del aquenio, denominadas discriminantes (Cuadro 1) para la agrupación de las accesiones, reflejan variabilidad morfológica en las poblaciones de $T$. filifolia del centro-sur de México. La longitud de la arista del aquenio no resultó de importancia para la diferenciación de los 5 grupos morfológicos, como lo fue en el trabajo previo en el que se identificaron 6 grupos (Serrato-Cruz y Barajas-Pérez, 2006). La forma, alargada o ancha, y el tamaño, grande o pequeño, de los aquenios de $T$. filifolia posiblemente se relacionen con procesos de dispersión por viento, penetración y almacenaje en el suelo, como sucede en otras especies silvestres herbáceas anuales (Thompson, 1987; Grime et al., 1988). El tamaño grande de semilla permite formar una reserva de semillas permanente en el suelo (Thompson, 1987), y un tamaño pequeño de la semilla se relaciona con una forma más compacta, lo cual puede favorecer el enterramiento de las semillas, escapar de los depredadores y poseer mecanismos de letargo de la germinación (Thompson, 1987; Grime et al., 1988). El tamaño de la semilla y su forma también le confieren un mejor desplazamiento, por ejemplo un tamaño pequeño y forma alargada de la semilla favorecen su desplazamiento dentro del perfil del suelo (Chambers et al., 1991) o en laderas. La relación específica entre características físicas del suelo donde se encontraron las plantas de T. filifolia de las que se colectaron aquenios podría ayudar a elucubrar más sobre la función de la forma y tamaño de estas estructuras reproductivas.

La distancia de Mahalanobis permitió concluir que los 5 grupos encontrados en el análisis de agrupación fueron morfológicamente diferentes; sin embargo, la prueba de resubstitución indicó inconsistencias en algunas accesiones que podrían pertenecer a otro grupo por presentar características intermedias entre grupos, lo que explicaría el parecido de algunos materiales principalmente en los Grupos I, III y V. No obstante que la mayoría de accesiones presentaron aquenios pequeños y anchos (a), pocas de ellas con aquenios grandes y delgados (b) y accesiones morfológicamente ubicables entre a y b (Cuadro 2, Fig. 4), se desconoce la dirección de esos cambios y las causas precisas asociadas con ellos. 
Cuadro 3. Distribución de colectas de T. filifolia (por grupo morfológico detectado en el análisis de agrupamiento, expresado en porcentaje) según origen en la Región Centro-Sur de México

\begin{tabular}{|c|c|c|c|c|c|c|c|c|c|c|}
\hline \multirow{2}{*}{ Grupo } & \multirow{2}{*}{ Colectas } & \multicolumn{9}{|c|}{ Origen } \\
\hline & & Gro. & Jal. & Méx. & Mich. & Mor. & Oax. & Pue. & $S L P$ & Ver: \\
\hline I & $\begin{array}{l}54,75,41,43,48,85,6,102,84,90,55,103,1 \\
5,49,76,80,12,97,73,8,9,11,21,66,67,10 \\
88,52,83,89,19,104,7,50,53,287,65,5,51 \\
, 82,42\end{array}$ & 2 & 10 & 5 & 33 & 0 & 14 & 12 & 2 & 22 \\
\hline II & $94,98,25,31,33,56,44,64,62,58,77,74,59$ & 15 & 0 & 8 & 46 & 23 & 8 & 0 & 0 & 0 \\
\hline III & $\begin{array}{l}20,57,63,45,71,1,23,39,68,14,99,46,96,86 \\
, 100,26,92,81,93,13,78,4,47,27,37\end{array}$ & 4 & 8 & 8 & 28 & 20 & 8 & 12 & 0 & 12 \\
\hline IV & $\begin{array}{l}16,17,61,72,24,30,32,34,3,95,22,91,40,18 \\
, 70,28,29,101,36,35\end{array}$ & 5 & 15 & 10 & 10 & 40 & 0 & 15 & 0 & 5 \\
\hline $\mathrm{V}$ & $38,60,69,79$ & 0 & 0 & 0 & 50 & 25 & 25 & 0 & 0 & 0 \\
\hline
\end{tabular}

Cuadro 4. Número de observaciones y porcentaje de colectas de T. filifolia de la región centro-sur de México, reclasificados en grupos mediante el método de resubstitución

\begin{tabular}{lllllll}
\hline Grupos & $I$ & $I I$ & $I I I$ & $I V$ & $V$ & Total \\
\hline I & 38 & 1 & 1 & 2 & 0 & 42 \\
$\%$ & 90.48 & 2.38 & 2.38 & 4.76 & 0 & 100 \\
II & 0 & 13 & 0 & 0 & 0 & 13 \\
$\%$ & 0 & 100 & 0 & 0 & 0 & 100 \\
III & 2 & 0 & 22 & 1 & 0 & 25 \\
$\%$ & 8 & 0 & 88 & 4 & 0 & 100 \\
IV & 0 & 0 & 0 & 20 & 0 & 20 \\
$\%$ & 0 & 0 & 0 & 100 & 0 & 100 \\
V & 0 & 0 & 1 & 0 & 3 & 4 \\
$\%$ & 0 & 0 & 25 & 0 & 75 & 100 \\
Total & 40 & 14 & 24 & 23 & 3 & 104 \\
$\%$ & 38.46 & 13.46 & 23.08 & 2.12 & 2.88 & 100 \\
\hline
\end{tabular}

En filas se presentan el número y la proporción de las accesiones ubicadas originalmente en el grupo y en columnas el grupo al que debería pertenecer. 


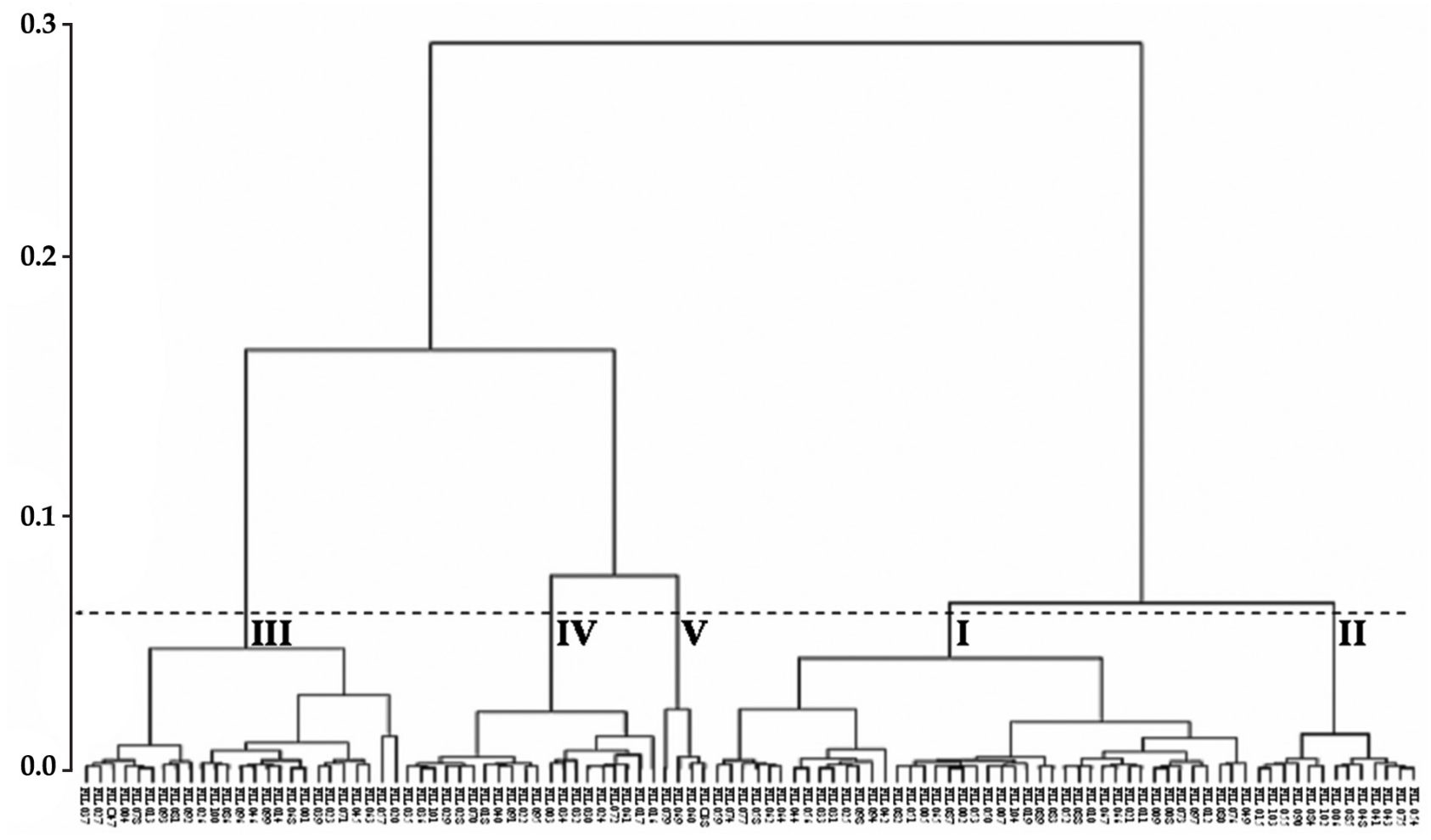

Figura 3. Dendrograma de 104 accesiones de Tagetes filifolia mediante caracteres morfométricos de aquenios utilizando la distancia euclidiana cuadrada y el método de aglomeración de Ward.

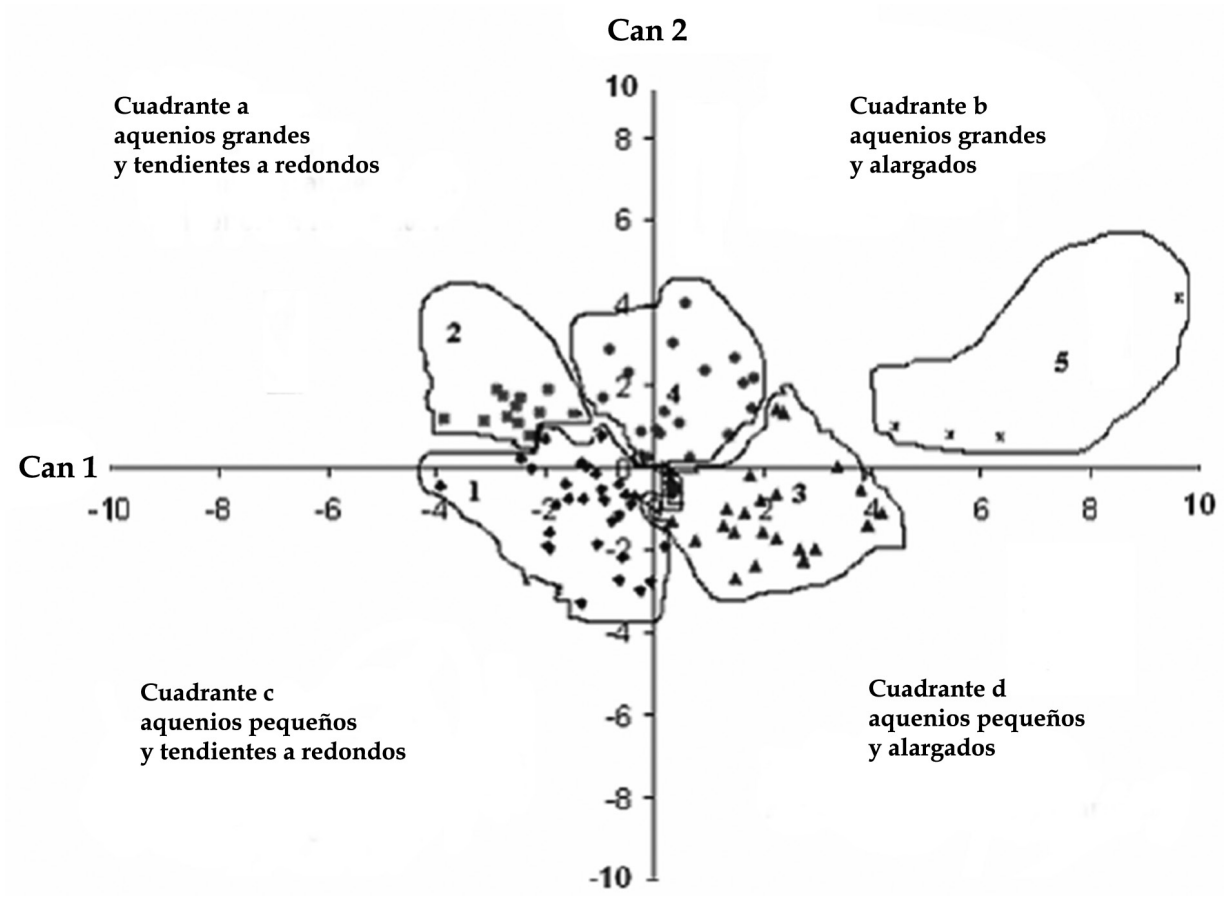

Figura 4. Representación bidimensional de los 5 grupos obtenidos en el análisis de agrupación de 104 accesiones de Tagetes filifolia en las 2 primeras funciones canónicas. 
Cuadro 5. Distancias Mahalanobis entre grupos formados con características de aquenios de T. filifolia obtenidos a partir del dendrograma mediante la distancia euclidiana y el método de Ward

\begin{tabular}{ccccc}
\hline Grupo & $I$ & $I I$ & $I I I$ & $I V$ \\
\hline II & $13.042^{* * *}$ & & & \\
III & $13.160^{* * *}$ & $28.883^{* * *}$ & & \\
IV & $10.902^{* * *}$ & $17.937^{* * *}$ & $13.413^{* * *}$ & \\
V & $70.214 * * *$ & $90.710^{* * *}$ & $39.473^{* * *}$ & $49.582^{* * *}$ \\
\hline
\end{tabular}

*** Significativo con $P \leq 0.001$.

La especie prospera en una amplia diversidad ambiental en el centro-sur de México (Apéndice 1), que hace complejo establecer la relación entre el ambiente y las características del aquenio; otras características de la planta como el periodo de floración, el crecimiento vegetativo, las cavidades oleríferas o el área foliar, entre otras, probablemente podrían tener una relación más clara con la diversidad ambiental. Al respecto, los gradientes de temperatura y humedad donde T. filifolia está adaptada son muy amplios (Neher, 1965; Serrato-Cruz y Barajas-Pérez, 2006). Un análisis de la variación morfológica del aquenio por transectos altitudinales, climáticos y edáficos en áreas específicas aclararía preguntas sobre la adaptación de $T$. filifolia en el centro-sur de México, como se ha realizado para otras plantas de la familia Asteraceae (Flann et al., 2002; Fici y Presti, 2003) y en otras especies (Dobzhansky, 1970; Murray et al., 2003; Yamada y Miyaura, 2005).

La medición digitalizada de las características de la forma y tamaño de los aquenios de T. filifolia provenientes de la región centro-sur de México mostró que hay gran variabilidad en su expresión morfológica agrupada en 5 morfotipos; así, la información generada en el presente estudio da precisión a la descripción publicada por Serrato-Cruz y Barajas-Pérez (2006) sobre este órgano reproductivo. La diversidad ambiental de la región CentroSur, en la que se expresa la variabilidad en los aquenios de T. filifolia, es amplia (Apéndice 1), pero no todos los grupos morfológicos identificados estuvieron distribuidos en toda esta región. El grupo $\mathrm{V}$ con aquenios grandes y muy delgados tiene una distribución restringida.

\section{Agradecimientos}

A los árbitros anónimos y a la Dra. Teresa Terrazas por la excelente edición y los atinados comentarios que ayudaron a enriquecer este manuscrito.

\section{Literatura citada}

Camarillo-de la R., G., L. D. Ortega-A., M. A. Serrato-Cruz, C. Rodríguez-H. y S. Barajas-P. 2007. Toxicidad del anisillo (Tagetes filifolia Lag.) en moscas blancas (Trialeurodes vaporariorum West.) en invernadero. Entomología Mexicana 6:1038-1043.

Chambers, J. C., J. A. MacMahon y J. H. Haefner. 1991. Seed entrapment in alpine ecosystems: effect of soil particle size and diaspore morphology. Ecology 72:1668-1677.

Dobzhansky, T. 1970. Genetics of the evolutionary process. Columbia University Press, New York. 505 p.

Fici, S. y R. M. L. Presti. 2003. Variation in the Senecio aethnensis group (Asteraceae) along an altitudinal gradient. Plant Biosystem 137:305-312.

Flann, C., P. Ladiges y N. G. Walsh. 2002. Morphological variation in Leptorhynchos squamatus (Gnaphalieae: Asteraceae). Australian Systematic Botany 15:205-219.

González-Andrés, F. y J. M. Ortiz. 1995. Seed morphology of Cytisophyllum, Cytisus, Chamaecytisus and Genista (Fabaceae: Genistaceae) species for characterization. Seed Science and Technology 23:289-300.

Grime, J. P., J. G. Hodgson y R. Hunt. 1988. Comparative plant ecology: a functional approach to common British species. Castlepoint, London. 752 p.

Johnson, D. E. 1998 Métodos multivariados aplicados al análisis de datos. Internacional Thompson, México, D.F. 566 p.

Murray, B. R., A. H. D. Brown y J. P. Grace. 2003. Geographic gradients in seed size among and within perennial Australian Glycine species. Australian Journal of Botany 51:47-56.

Neher, R. T. 1965. Monograph of the genus Tagetes (Compositae). Indiana University Press, Bloomington. $1004 \mathrm{p}$.

Nieto-Ángel, R., S. A. Pérez-Ortega, C. A. Núñez-Colín, J. Martínez-Solís y F. González-Andrés 2009. Seed and endocarp traits as markers of the biodiversity of regional sources of germplasm of tejocote (Crataegus spp.) from central and southern Mexico. Scientia Horticulturae 121:166170. 
Reyes-T., B., J. C. Salazar-T. y A. Juárez-Estudillo. 2007. Uso de extractos orgánicos y acuosos en el control del pulgón Eriosoma crataegi Oestlund en tejocote (Crataegus pubescens H. B. K.). Entomología Mexicana 6:1044-1048.

SAS Institute.1999. SAS/STAT software: changes and enhancements through release 8. SAS Institute, Cary, North Carolina.

Serrato-Cruz, M. A. y J. S. Barajas-Pérez. 2006. Poblaciones silvestres de de Tagetes filifolia Lag. en el centro y sur de México. Revista Fitotecnia Mexicana 29 (número especial 2):7-12.

Serrato-Cruz, M. A., S. Miranda-Colín, A. García-Velázquez. y F. Castillo-García. 1998. Aislamiento reproductivo en plantas de cempoalxóchitl (Tagetes spp). Revista Fitotecnia Mexicana 21:127-138.

Serrato C., M. A. y L. Quijano A. 1994. Usos de algunas especies de Tagetes Revisión bibliográfica (1984-1992). Memorias I Simposium Internacional y II Reunión Nacional sobre Agricultura Sostenible Tradicional. CEICADAR. Colegio de Posgraduados. Puebla. p. 228-238.

Serrato C., M. A., B. Reyes, L. Ortega, A. Domingo, N. Gómez,
F. López, M. Sánchez, L. Carvajal, O. Jiménez, A. Morgado, E. Pérez, J. Quiroz y C. I. Vallejo. 2003. Anisillo (Tagetes filifolia Lag.): Recurso genético mexicano para controlar la mosquita blanca (Bemisia sp. y Trialeurodes sp.). Revista del Jardín Botánico Nacional 24:65-70.

Thompson, K. 1987. Seeds and seed banks. New Phytologist 106:23-34.

Turner, B. L. 1996. The Comps of Mexico: a systematic account of the family Asteraceae, vol. 6, Tageteae and Anthemideae. Phytologia Memoirs 10:1-93.

Villarreal Q., J. A. 2003. Familia Compositae. Tribu Tageteae. Flora del Bajío y de regiones adyacentes. Fascículo 113. Instituto de Ecología, Pátzcuaro, Michoacán. 85 p.

Ward, J. H. Jr. 1963. Hierachical grouping to optimize an objetive function. Journal of the American Statistical Association 58:236-244.

Wilcox, D., B. Dove, D. Mcdavid y D. Greer. 1995. Image tool, version 3. Users' guide. University of Texas Health Science Center, San Antonio. . 62 p.

Yamada H. y T. Miyaura. 2005. Geographic variation in nut size of Castanopsis species in Japan. Ecological Research 20:3-9.

Apéndice 1. Origen de las 104 recolecciones de T.filifolia obtenidas en la región centro-sur de México, 2004.

\begin{tabular}{|c|c|c|c|}
\hline Colecta & Origen & $\begin{array}{l}\text { Coordenadas } \\
\text { (latitud N, longitud O) }\end{array}$ & Altitud \\
\hline FIL 001 & San Antonio Ocopetatlán, Puebla & Donación & NR \\
\hline FIL 002, 003 & $\begin{array}{l}\text { Colonia Manuel González, Zentla, } \\
\text { Veracruz }\end{array}$ & Donación & NR \\
\hline FIL 004 & Atexca, Zacatlán, Puebla & Donación & NR \\
\hline FIL 005 & Ixcatlo, Ixhuatlán, Veracruz & Donación & NR \\
\hline FIL 006, 007, 008, 009 & Xoxonacatla, Zacatlán, Puebla & Donación & NR \\
\hline FIL 010, 011 & Sayula, Jalisco & $19^{\circ} 49.694^{\prime} 103^{\circ} 37.610^{\prime}$ & 1807 \\
\hline FIL 012, 013 & San Antonio, San Gabriel, Jalisco. & $19^{\circ} 47.812^{\prime} 103^{\circ} 53.277^{\prime}$ & 999 \\
\hline FIL 014, 015 & Los Parajes, Cocula, Jalisco & $20^{\circ} 22.519^{\prime} 102^{\circ} 28.721^{\prime}$ & 1518 \\
\hline FIL 016, 017, 018 & Atotonilco, Jalisco & $20^{\circ} 27.059^{\prime} 102^{\circ} 32.784^{\prime}$ & 1722 \\
\hline FIL 019 & Encinal, Totutla, Veracruz. & $19^{\circ} 12.732^{\prime} 96^{\circ} 49.740^{\prime}$ & 846 \\
\hline FIL 020 & Úrsulo Galván, Coatepec, Veracruz & $19^{\circ} 27.138^{\prime} 96^{\circ} 57.632^{\prime}$ & 1184 \\
\hline FIL 021 & Acajete, La Joya, Veracruz & $19^{\circ} 06.724^{\prime} 97^{\circ} 01.929^{\prime}$ & 2187 \\
\hline $\begin{array}{l}\text { FIL } 022,023,024,025,026,027 \text {, } \\
028,029\end{array}$ & Yecapixtla, Morelos & $18^{\circ} 53.040^{\prime} 98^{\circ} 51.996^{\prime}$ & 1558 \\
\hline FIL 030, 031, 032, 033, 034 & Nepantla, Morelos & $18^{\circ} 58.611^{\prime} 98^{\circ} 50.380^{\prime}$ & 1980 \\
\hline FIL 035 & Zacatlán, Puebla & $19^{\circ} 57.044^{\prime} 98^{\circ} 03.310^{\prime}$ & 2567 \\
\hline
\end{tabular}


Apéndice 1. Continúa.

\begin{tabular}{|c|c|c|c|}
\hline Colecta & Origen & $\begin{array}{l}\text { Coordenadas } \\
\text { (latitud } N \text {, longitud O) }\end{array}$ & Altitud \\
\hline FIL 036 & Zacatlán, Puebla & $20^{\circ} 00.181^{\prime} 98^{\circ} 05.888^{\prime}$ & 2404 \\
\hline FIL 037, 038, 039, 040 & Ecatzingo, Morelos & $18^{\circ} 58.150^{\prime} 98^{\circ} 45.104^{\prime}$ & 2557 \\
\hline FIL 041 & Pátzcuaro, Michoacán & $19^{\circ} 20.099^{\prime} 101^{\circ} 42.291^{\prime}$ & 2153 \\
\hline FIL 042 & Tinbangato, Michoacán & $19^{\circ} 29.500^{\prime} 101^{\circ} 52.568^{\prime}$ & 1893 \\
\hline FIL 043, 044, 045 & Uruapan, Michoacán & $19^{\circ} 20.059^{\prime} 102^{\circ} 04.742^{\prime}$ & 1454 \\
\hline FIL 046, 047, 048 & Charapendo, Uruapan, Michoacán & $19^{\circ} 15.648^{\prime} 102^{\circ} 03.313^{\prime}$ & 973 \\
\hline FIL 049, 050, 051 & Tancítaro, Michoacán & $19^{\circ} 21.185^{\prime} 102^{\circ} 22.505^{\prime}$ & 2066 \\
\hline FIL 052, 053, 054 & Peribán, Michoacán & $19^{\circ} 33.123^{\prime} 102^{\circ} 26.668^{\prime}$ & 1449 \\
\hline FIL 055, 056, 057, 058 & Los Reyes, Michoacán & $19^{\circ} 37.756^{\prime} 102^{\circ} 29.325^{\prime}$ & 1308 \\
\hline FIL 059, 060, 061 & Tinguindin, Michoacán & $19^{\circ} 48.935^{\prime} 102^{\circ} 30.725^{\prime}$ & 1802 \\
\hline FIL 062, 063, 064 & Santo Tomás Chilchota, Michoacán & $19^{\circ} 48.934^{\prime} 102^{\circ} 30.726^{\prime}$ & 1780 \\
\hline FIL 065 & Cheranastico, Chilchota, Michoacán & $19^{\circ} 48.934^{\prime} 102^{\circ} 30.726^{\prime}$ & 2154 \\
\hline FIL 066 & Paracho, Michoacán & $19^{\circ} 48.934^{\prime} 102^{\circ} 30.726^{\prime}$ & 2286 \\
\hline FIL 067, 068, 069 & San Ángel Ziracuaretiro, Michoacán & $19^{\circ} 27.647^{\prime} 101^{\circ} 53.168^{\prime}$ & 1719 \\
\hline FIL 070 & El Temascal, Morelia, Michoacán & $19^{\circ} 39.087100^{\circ} 57.245^{\prime}$ & 2178 \\
\hline FIL 071 & Lindavista, Zitácuaro, Michoacán & $19^{\circ} 39.088100^{\circ} 57.243^{\prime}$ & 1653 \\
\hline FIL 072, 073, 074 & El Cinco, Valle De Bravo, México & $19^{\circ} 24.951^{\prime} 100^{\circ} 09.262^{\prime}$ & 2648 \\
\hline FIL 075 & Zacapoaxtla, Puebla & $19^{\circ} 55.778^{\prime} 97^{\circ} 35.693^{\prime}$ & 1680 \\
\hline FIL 076 & Cd. Cárdenas, San Luis Potosí & $\begin{array}{l}\text { Mercado Municipal de Cd. Valles, } \\
\text { SLP }\end{array}$ & $\begin{array}{l}\dagger 1200 \\
1500\end{array}$ \\
\hline FIL 077 & Ayutla, Oaxaca. & $16^{\circ} 59.098^{\prime} 96^{\circ} 08.574^{\prime}$ & 1902 \\
\hline FIL 078 & Yacochi, Oaxaca, & $17^{\circ} 08.675^{\prime} 96^{\circ} 02.816^{\prime}$ & 2361 \\
\hline FIL 079 & Yacochi, Oaxaca, & $17^{\circ} 07.340^{\prime} 96^{\circ} 02.326^{\prime}$ & 2363 \\
\hline FIL 080 & Miahuatlán, Oaxaca & $16^{\circ} 13.798^{\prime} 96^{\circ} 31.557^{\prime}$ & 2361 \\
\hline FIL 081 & Ciénega, Oaxaca & $16^{\circ} 12.538^{\prime} 96^{\circ} 32.116^{\prime}$ & 2388 \\
\hline FIL 082 & Paxtlán, Oaxaca. & $16^{\circ} 10.271^{\prime} 96^{\circ} 30.166^{\prime}$ & 2476 \\
\hline FIL 083 & Loxicha, Oaxaca & $16^{\circ} 07.887^{\prime} 96^{\circ} 29.794^{\prime}$ & 2686 \\
\hline FIL 084 & Sotavento, Sierra Madre del Sur & $16^{\circ} 04.893^{\prime} 96^{\circ} 28.060^{\prime}$ & 2384 \\
\hline FIL 085 & Sotavento, Sierra Madre del Sur & $15^{\circ} 59.515^{\prime} 96^{\circ} 31.518^{\prime}$ & 1543 \\
\hline FIL 086 & Tequila, Zongolica, Veracruz & $18^{\circ} 43.751^{\prime} 97^{\circ} 03.849^{\prime}$ & 1993 \\
\hline FIL 087 & Tequila, Zongolica, Veracruz & $18^{\circ} 43.236^{\prime} 97^{\circ} 03.808^{\prime}$ & 1830 \\
\hline FIL 088 & Ayoxapan, Zongolica, Veracruz & $18^{\circ} 40.074^{\prime} 96^{\circ} 57.590^{\prime}$ & 1453 \\
\hline
\end{tabular}


Apéndice 1. Continúa.

\begin{tabular}{|c|c|c|c|}
\hline Colecta & Origen & $\begin{array}{l}\text { Coordenadas } \\
\text { (latitud } N \text {, longitud } O \text { ) }\end{array}$ & Altitud \\
\hline FIL 089 & Zongolica, Veracruz & $18^{\circ} 40.612^{\prime} 96^{\circ} 56.157^{\prime}$ & 1004 \\
\hline FIL 090 & Acajete, Veracruz & $19^{\circ} 34.864^{\prime} 96^{\circ} 59.513^{\prime}$ & 1824 \\
\hline FIL 091 & Temazcaltepec, México & $19^{\circ} 09.997^{\prime} 99^{\circ} 53.599$ & 2674 \\
\hline FIL 092 & Temazcaltepec, México & $19^{\circ} 09.997^{\prime} 99^{\circ} 59.398$ & 2155 \\
\hline FIL 093 & Tejupilco, México & $18^{\circ} 55.231^{\prime} 100^{\circ} 08.907$ & 1427 \\
\hline FIL 094, 095 & $\begin{array}{l}\text { El Aserradero, Coyuca de Catalán, } \\
\text { Guerrero }\end{array}$ & $17^{\circ} 58.839^{\prime} 101^{\circ} 13.746^{\prime}$ & 1898 \\
\hline FIL 096 & $\begin{array}{l}\text { El Aserradero, Coyuca de Catalán, } \\
\text { Guerrero }\end{array}$ & $17^{\circ} 58.839^{\prime} 101^{\circ} 13.746^{\prime}$ & 1612 \\
\hline FIL 097, 098 & $\begin{array}{l}\text { El Aserradero, Coyuca de Catalán, } \\
\text { Guerrero }\end{array}$ & $17^{\circ} 58.839^{\prime} 101^{\circ} 13.746^{\prime}$ & 1403 \\
\hline FIL 099 & Naolinco, Veracruz & $18^{\circ} 43.775^{\prime} 97^{\circ} 04.024^{\prime}$ & 1505 \\
\hline FIL 100 & $\begin{array}{l}\text { Colonia Manuel González, Zentla, } \\
\text { Veracruz }\end{array}$ & Donación & NR \\
\hline FIL 101 & Jilotzingo, Zacatlán, Puebla. & Donación & NR \\
\hline FIL 102 & San Martín Tlacolula, Veracruz. & Donación & NR \\
\hline FIL 103 & Santa Maria Ocotlán, Putla Oaxaca & Donación & NR \\
\hline FIL 104 & Tlalámac, Ozumba, México. & $19^{\circ} 05^{\prime} 98^{\circ} 49^{\prime}$ & 1780 \\
\hline
\end{tabular}

†Altitud probable; NR, no se registró. 
